[4] S. Odén, Nova Acta Regiae Soc. Sci. Upsaliensis Ser. IV 3 (1913) Nr. 4 Z. Phys. Chem. Stöchiom. Verwandtschaftsl. 80 (1912) 709.

[5] E. Weitz, K. Gieles, J. Singer, B. Alt, Chem. Ber. 89 (1956) 2365

[6] J. Weiß, M. Göbl, Fresenius Z. Anal. Chem. 320 (1985) 439.

[7] R. Steudel, G. Holdt, J. Chromatogr. 36 I (1986) 379.

[8] R. Steudel, R. Strauss, D. Jensen, Chem-Ztg. 109 (1985) 349.

[9] Chromatograph Varian 5000 mit mikrocomputergesteuerter Gradientenbildung, Rheodyne-Schleifeninjektor $(10 \mu \mathrm{L})$, Chrompack-CartridgeGlassäule (CT ${ }^{\mathrm{tm}}$ SpherC18; $\left.l=10 \mathrm{~cm}\right)$, Varian UV5-Detektor $(215 \mathrm{~nm})$ Hewlett-Packard-3390A-Integrator, Knauer-Schreiber. Weitere experi mentelle Einzelheiten siehe [7]

[10] Siehe z. B. M. Eccleston, D. P. Kelly, J. Bacteriol. 134 (1978) 718, zit. Lit.

[11] P. A. Trudinger, Rev. Pure Appl. Chem. 17 (1967) 1.

[12] D. P. Kelly, Philos. Trans. R. Soc. London Ser. B 298 (1982) 499, Microbiol. Sci. 2 (1985) 105

[13] W. Hazeu, W. Bijleveld, J. T. C. Grotenhuis, E. Kakes, J. G. Kuenen, Antonie van Leeuwenhoek, im Druck; die Bakterien wurden in Delft von $\boldsymbol{W} . \boldsymbol{H}$. kultiviert und für die Oxidations- und Extraktionsexperimente per Eilpost nach Berlin transportiert.

[14] R. Steudel, H.-J. Mäusle, D. Rosenbauer, H. Möckel, T. Freyholdt, $A n$ gew. Chem. 93 (1981) 402; Angew. Chem. Int. Ed. Engl. 20 (1981) 394; R. Strauss, R. Steudel, Fresenius Z. Anal. Chem., im Druck.

[15] R. E. Davis, J. Am. Chem. Soc. 80 (1958) 3565; R. Steudel, H.-J. Măusle, Z. Anorg. Allg. Chem. 457 (1979) 165.

[16] P. A. Trudinger, Aust. J. Biol. Sci. $/ 7$ (1964) 446

[17] M. Schmidt, Z. Anorg. Allg. Chem. 289 (1957) 158.

[18] Bei denjenigen phototrophen und chemolithotrophen Schwefelbakterien, die ebenfalls „ „S ${ }^{0}$ globules“ bilden (z. T. extra-, z. T. intrazellulär), können wahrscheinlich statt der Polythionate auch andere Verbindungen mit polaren Gruppen die Oberfläche des hydrophoben $S_{8}$-Trŏpf chens bedecken und dadurch hydrophil machen; vgl. hierzu die Stabilisierung von Schwefelpartikeln in Wasser durch Adsorption organischer Carbonsäuren (z. B. Polyacrylsãure): A. Asanov, A. Ilyasov, K. S. Akhmedov, Dokl. Akad. Nauk UzSSR 1985, 35 (Chem. Abstr. 104 (1986) 136611), zit. Lit.

[19] J. Boulege, Phosphorus Sulfur 5 (1978) 127.

[20] G. W. Donaldson, F. J. Johnston, J. Phys. Chem. 73 (1969) 2064; R. H. Dinegar, R. H. Smellie, J. Colloid Sci. 7 (1952) 370.

\section{Stereoselektive Arenalkylierung mit}

\section{Threonin-trifluormethansulfonaten ${ }^{* *}$}

\section{Von Franz Effenberger* und Thomas Weber \\ Professor Rudolf Gompper zum 60. Geburtstag gewidmet}

Alkyltrifluormethansulfonate (Alkyltriflate) wurden bisher nur vereinzelt zur Alkylierung von Arenen eingesetzt ${ }^{[1]}$. Wir konnten jetzt die $N$-geschützten Triflate von $(S)$ - und (R)-Serin-methylester sowie die $N$-geschützten Triflate 1 aller diastereomeren Threonin-methylester in sehr guten Ausbeuten und unter vollständiger Erhaltung der Konfiguration darstellen. Dazu wurden die $N$-Phthaloyl-geschützten Aminosäureester mit Trifluormethansulfonsäureanhydrid in Gegenwart von Pyridin umgesetzt. Mit den Triflaten der Serin- bzw. der Threoninester können Benzol und Benzolderivate alkyliert werden, wobei Phenylalaninbzw. $\beta$-Methyl-phenylalaninester 2 erhalten werden.

Diese Reaktionen verlaufen unter den von uns angewendeten Bedingungen in allen Fällen unter Erhaltung der Konfiguration an C-2 der Aminosăureester. Von Interesse, aber schwierig vorauszusagen, war die Stereochemie an C-3 der $\beta$-Methyl-phenylalaninester 2. Die Friedel-CraftsAlkylierung von Arenen mit optisch aktiven Alkylierungs-

[*] Prof. Dr. F. Effenberger, Dipl.-Chem. T. Weber Institut für Organische Chemie der Universităt Pfaffenwaldring 55, D-7000 Stuttgart 80

[**] Zur elektrophilen Aromatensubstitution, 32. Mitteilung. Diese Arbeit wurde von der Deutschen Forschungsgemeinschaft und dem Fonds der Chemischen Industrie gefördert. $T$. $W$. dankt dem Fonds der Chemischen Industrie für ein Doktorandenstipendium. - 31. Mitteilung: F. Effenberger, F. Reisinger, K. H. Schönwălder, P. Băuerle, J. J. Stezowski, K. H. Jogun, K. Schöllkopf, W. D. Stohrer, J. Am. Chem. Soc., im Druck. mitteln findet im allgemeinen unter weitgehender Racemisierung statt ${ }^{[2]}$. Insbesonders Suga et al. haben jedoch gezeigt, daß Alkylierungen mit optisch aktiven Verbindungen auch weitgehend racemisierungsfrei verlaufen können, wenn bestimmte strukturelle Voraussetzungen gegeben $\operatorname{sind}^{[3]}$. In der Regel kommt es bei diesen Alkylierungen zur Inversion der Konfiguration am angreifenden C-Atom des Alkylierungsagens. Retention wurde bei der Alkylierung mit $\beta$-Phenylalkylhalogeniden beobachtet: Im ersten Schritt entsteht durch intramolekulare Alkylierung unter Inversion eine Cyclohexadienylium-Zwischenstufe, die im zweiten Schritt, wiederum unter Inversion, mit dem Aren reagiert ${ }^{[4]}$.

Bei der Alkylierung von Benzol mit den Triflaten der $N$ Phthaloyl-threonin- 1a,b und $N$-Phthaloyl-allothreoninmethylester 1c,d haben wir in allen Fällen die $\beta$-Methyl$N$-phthaloyl-phenylalanin-methylester 2 erhalten. Die Gesamtausbeuten an 2 liegen nur bei $20-40 \%$, da neben der Substitution von $1 \mathrm{zu} 2$ in starkem Maße auch Eliminierung $\mathrm{zu} \alpha, \beta-(25-30 \%)$ und $\beta, \gamma$-Dehydroaminosäureestern (35-40\%) stattfindet.

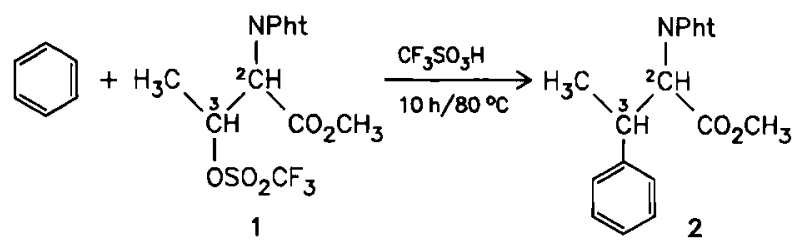<smiles>CC(CN=P)OS(=O)(=O)OC(F)(F)F</smiles>

3<smiles>CCOC(=O)O[C@@H](C)CCOC(F)(F)F</smiles>

(S) -4<smiles>O=C1CC(=O)c2ccccc21</smiles>

Tabelle 1. Konfigurationsănderungen bei der Benzolalkylierung mit den dia stereomeren Threonin-Derivaten 1 zu Phenylalanin-Derivaten 2 [a]

\begin{tabular}{lllll}
\hline & Edukt & & Produkte & $\begin{array}{l}\text { Ausbeute- } \\
\text { verhältnis }\end{array}$ \\
\hline 1a & $(2 S, 3 R)$ & 2a & $(2 S, 3 S)+2 \mathrm{c}$ & $97: 3$ \\
1b & $(2 R, 3 S)$ & 2b & $(2 R, 3 R)+2 \mathrm{~d}$ & $98: 2$ \\
1c & $(2 S, 3 S)$ & 2c & $(2 S, 3 R)+2 \mathrm{a}$ & $40: 60$ \\
1d & $(2 R, 3 R)$ & 2d & $(2 R, 3 S)+2 \mathrm{~b}$ & $40: 60$ \\
\hline
\end{tabular}

[a] $3 R$ in 1 und $3 S$ in 2 kennzeichnen die gleiche Konfiguration an C-3.

Während die Konfiguration an C-2 - wie schon erwähnt bei den Umsetzungen von 1a-d zu 2 erhalten bleibt, verhalten sich die Threonin- 1a,b und Allothreonin-Derivate 1c,d bei dieser Alkylierung bezüglich der Stereochemie an C-3 sehr unterschiedlich (Tabelle 1).

Die Threonin-Derivate 1a,b reagieren unter weitgehender Retention an C-3, während die Allothreonin-Derivate 1c,d zu Diastereomerengemischen im Verhältnis $2: 3$ führen. Die Charakterisierung und Zuordnung der vier Diastereomere 2a-d erfolgte durch Vergleich der Drehwerte und der ${ }^{1} \mathrm{H}$-NMR-Daten mit Literaturwerten ${ }^{[5]}$. Für 2a haben wir die Konfiguration durch eine Röntgen-Strukturuntersuchung gesichert ${ }^{[6]}$. Darüber hinaus konnten wir zeigen, daß das durch saure Esterhydrolyse, Entfernung der Phthaloyl-Schutzgruppe und Acetylierung aus 2a erhaltene Produkt mit dem beschriebenen $(2 S, 3 S)-N$-Acetyl- $\beta$ methyl-phenylalanin ${ }^{[7]}$ identisch ist.

Die hohe Stereoselektivität der Alkylierung von Benzol mit 1a,b und das davon abweichende Verhalten von $1 c, d$ 
erklären wir mit den unterschiedlichen Konformationen der als Zwischenstufen der Alkylierung auftretenden Carbenium-Ionen $B$ und $D$.

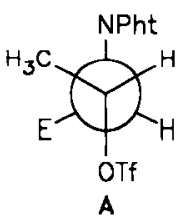
A

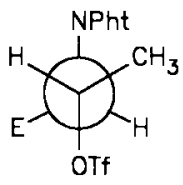

C
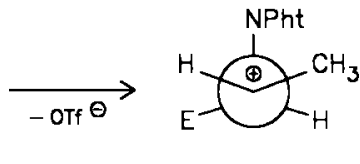

D<smiles>C1CO[GeH2]C1</smiles>

$\mathrm{E}=\mathrm{CO}_{2} \mathrm{CH}_{3} ;$ Tf $=\mathrm{SO}_{2} \mathrm{CF}_{3}$

Benzol wird mit $(S)$ - und $(R)$-1-Methyl-2-phthaloylaminoethyltriflat 3 unter teilweiser Retention, mit (S)-3-Trifluormethansulfonyloxy-buttersäure-methylester $(S)-4 \mathrm{da}-$ gegen unter vollständiger Racemisierung alkyliert. Daraus schließen wir, daß für den Dissoziationsschritt der Threonin-Derivate 1a,b die Konformation A, für den der Allothreonin-Derivate 1c,d die Konformation $\mathbf{C}$ bevorzugt ist. In dem aus A gebildeten Carbenium-Ion B ist eine Rotation durch die beiden benachbarten größeren Substituenten $\left(\mathrm{CH}_{3}, \mathrm{CO}_{2} \mathrm{CH}_{3}\right)$ erschwert, und das Aren kann - weil darüber hinaus das kationische Zentrum durch den $\mathrm{N}$ Phthaloyl-Rest von oben abgeschirmt wird - nur von der Unterseite her mit B reagieren; dies erklärt die Retention bei den Reaktionen von 1a,b. Für die kationische Zwischenstufe $\mathbf{D}$ dagegen ist keine oder nur eine geringe Rotationshinderung zu erwarten, was durch die Ergebnisse der Umsetzungen von 1c,d bestätigt wird.

Ähnlich wie Benzol lassen sich auch Alkyl- und Alkoxybenzole mit den Triflaten 1 der Threoninester alkylieren. Phenylalanine und $\beta$-Methyl-phenylalanine wie 2 können auch aus Arenen, den $\mathrm{N}$-geschützten Estern des Serins bzw. Threonins und Trifluormethansulfonsäureanhydrid im Eintopfverfahren (ohne Isolierung der Triflate vom Typ 1) synthetisiert werden. Trifluormethansulfonsäure läßt sich als Bariumsalz abscheiden und weitgehend zurückgewinnen.

\section{Arbeitsvorschriften}

2a: $1.58 \mathrm{~g}$ (4 mmol) $N$-Phthaloyl- $O$-(trifluormethansulfonyl)-( $2 S, 3 R$ )-threonin-methylester $1 \mathrm{a}$ und $0.61 \mathrm{~g}(4 \mathrm{mmol})$ Trifluormethansulfonsáure werden in $8 \mathrm{~mL}$ Benzol $10 \mathrm{~h}$ bei $80^{\circ} \mathrm{C}$ unter Feuchtigkeitsausschluß gerïhrt und anschließend im Vakuum von überschüssigem Benzol befreit. Nach Zusatz von Wasser zum Rückstand wird das Gemisch mit Chloroform extrahiert. Die vereinigten organischen Phasen werden nach Trocknen mit $\mathrm{MgSO}_{4}$ eingeengt: der Rückstand wird über Kieselgel mit Petrolether/Ethylacetat $9: 1$ chromatographiert. Ausbeute $300 \mathrm{mg}(24 \%) 2 \mathrm{a}, \mathrm{Fp}=107^{\circ} \mathrm{C}$ (Petrolether), $[\alpha]^{201}-139.1^{\circ}\left(c=1, \mathrm{CHCl}_{3}\right)$, und $10 \mathrm{mg}(0.77 \%) 2 \mathrm{c}$, Öl, $[\alpha]_{\mathrm{b}}^{20}-120.5^{\circ}$ $\left(c=0.6, \mathrm{CHCl}_{3}\right)$.

Eintopfverfahren: $0.53 \mathrm{~g}$ ( $2 \mathrm{mmol}) \mathrm{N}$-Phthaloyl-(2S,3R)-threonin-methylester und $0.56 \mathrm{~g}(2 \mathrm{mmol})$ Trifluormethansulfonsāureanhydrid werden wie vorstehend beschrieben umgesetzt. Ausbeute $0.15 \mathrm{~g}(23 \%) 2 \mathrm{a},[\alpha]_{0}^{20}-138.3^{\circ}(c=1$, $\mathrm{CHCl}_{3}$ ).

Eingegangen am 6. Oktober, veränderte Fassung am 17. November 1986 [Z 1947]

[1] a) F. Effenberger, Angew. Chem. 92 (1980) 147; Angew. Chem. Int. Ed. Engl. 19 (1980) 151, zit. Lit.; b) B. L. Booth, R. N. Haszeldine, K. Laali, $J$. Chem. Soc. Perkin Trans. I 1980, 2887.

[2] a) C. C. Price, M. Lund, J. Am. Chem. Soc. 62 (1940) 310; b) A. Streitwieser, Jr., P. J. Stang, ibid. 87 (1965) 4953.
[3] a) T. Nakajima, S. Suga, T. Sugita, K. Ichikawa, Bull. Chem. Soc. Jpn. 40 (1967) 2980 ; b) T. Nakajima, S. Masuda, S. Nakashima, T. Kondo, Y. Nakamoto, S. Suga, ibid. 52 (1979) 2377; c) O. Piccolo, F. Spreafico, G. Visentin, E. Valoti, J. Org. Chem. 50 (1985) 3945.

[4] M. Shinji, T. Nakajima, S. Suga, Bull. Chem. Soc. Jpn. 56 (1983) 1089.

[5] Y. Kataoka, Y. Seto, M. Yamamoto, T. Yamada, S. Kuwata, H. Watanabe, Bull. Chem. Soc. Jpn. 49 (1976) 1081.

[6] Prof. Dr. John J. Stezowski danken wir für die Röntgen-Strukturanalyse.

[7] a) H. Arold, M. Eule, S. Reissmann, Z. Chem. 9 (1969) 447; b) G. I. Tsuchihashi, S. Mitamura, K. Ogura, Bull. Chem. Soc. Jpn. 52 (1979) 2167 .

\section{Heteronucleare Polyhydrido-Komplexe mit Floßstruktur - ein Vergleich von Synthesen mit Kupfer- und Goldalkoxiden *}

Von Bruce R. Sutherland, Douglas M. Ho, John C. Huffman und Kenneth G. Caulton*

Gegenwärtig herrscht großes Interesse an heteronuclearen Metallkomplexen, welche sowohl Übergangs- als auch Münzmetalle enthalten; besondere Beachtung finden dabei Verbindungen mit Hydrido-Liganden ${ }^{[1,2]}$. Normalerweise werden solche Verbindungen durch Umsetzung von $\mathrm{ML}_{\mathrm{x}}^{\oplus}$-Komplexen $\left(\mathrm{M}=\mathrm{Cu}, \mathrm{Ag}, \mathrm{Au} ; \mathrm{L}=\mathrm{PPh}_{3}, \mathrm{CH}_{3} \mathrm{CN}\right)$ mit Metallhydriden hergestellt ${ }^{[3-5]}$.Kürzlich berichteten wir über ein Beispiel für eine alternative Synthesemethode: Bei der Reaktion von $[\mathrm{CuOtBu}]_{4}$ mit $\left[\mathrm{OsH}_{4}\left(\mathrm{PMe}_{2} \mathrm{Ph}\right)_{3}\right]$ entsteht unter Eliminierung von $t \mathrm{BuOH}$ der $\mathrm{Cu}_{3} \mathrm{Os}_{3}$-Cluster $\left[\mathrm{CuOsH}_{3}\left(\mathrm{PMe}_{2} \mathrm{Ph}\right)_{3}\right]_{3}{ }^{[6]}$. Wir haben nun die Reaktionen des zweikernigen Polyhydrids $\left[\mathrm{Re}_{2} \mathrm{H}_{8}\left(\mathrm{PMe}_{2} \mathrm{Ph}\right)_{4}\right] 2$ mit den $\mathrm{Cu}$ - und $\mathrm{Au}$-Komplexen $\left[\mathrm{Ph}_{3} \mathrm{PCuO} t \mathrm{Bu}\right]_{2} 1$ bzw. $\left[\mathrm{Ph}_{3} \mathrm{PAu}-\right.$ $\mathrm{O} t \mathrm{Bu}] 6^{[7]}$ vergleichend studiert.

$$
\begin{array}{r}
1 / 2\left[\mathrm{Ph}_{3} \mathrm{PCuO} t \mathrm{Bu}\right]_{2}+\left[\mathrm{Re}_{2} \mathrm{H}_{8}\left(\mathrm{PMe}_{2} \mathrm{Ph}\right)_{4}\right] \underset{\mathrm{C}_{h} \mathrm{H}_{\mathrm{h}}}{\mathbf{1}} \\
\quad \\
\quad 1 / 2\left[\mathrm{Re}_{2} \mathrm{CuH}_{7}\left(\mathrm{PMe}_{2} \mathrm{Ph}\right)_{4}\right]_{2}+t \mathrm{BuOH}+\mathrm{PPh}_{3}
\end{array}
$$
3

Die Reaktion von $\left[\mathrm{Ph}_{3} \mathrm{PCuO} t \mathrm{Bu}\right]_{2} \mathbf{1}^{[\mathrm{s}]}$ mit dem Rheniumkomplex 2 läuft schon während des Mischvorgangs ab [Gl. (a) $]^{[9]}$. Als dunkelbraunes Produkt wird nach Entfernen des Solvens und der flüchtigen Beiprodukte nahezu quantitativ der $\mathrm{Re}_{4} \mathrm{Cu}_{2}$-Komplex 3 isoliert. Bei der Reaktion entsteht weder $\mathrm{H}_{2}$ noch elementares Kupfer. Diese Befunde, das 'H-NMR-Spektrum und die Röntgen-Strukturanalyse an einem Kristall von $3^{[11]}$, der aus Toluol/Hexan isoliert wurde, sind strukturbeweisend. Der Tetradecahydrido-Komplex 3 ist bisher der hydridreichste, neutrale Cluster. Die sechs Metallatome bilden ein zentrosymmetrisches, nahezu planares Parallelogramm (Abb. 1); diese Anordnung erinnert an die Strukturen der Cluster 4 und 5.

$\left[\mathrm{Re}_{4} \mathrm{Cu}_{2} \mathrm{H}_{16}\left(\mathrm{PMe}_{2} \mathrm{Ph}\right)_{8}\right]^{2 \oplus} 4^{[12]}$

$\left.\left[\mathrm{Mn}_{4} \mathrm{Cu}_{2} \mathrm{H}_{16}(\mathrm{CO})_{12}(\mathrm{EtO})_{2} \mathrm{POP}(\mathrm{OEt})_{2}\right\}_{2}\right] \mathbf{5}^{[13]}$

Für die 14 Hydrido-Liganden in 3 existieren vier Bindungstypen: Sechs Hydrido-Liganden sind nur an Rhenium (zwei terminal, vier Re-Re-verbrückend) und acht sind so-

[*] Prof. Dr. K. G. Caulton, Dr. B. R. Sutherland, Dr. D. M. Ho, Dr. J. C. Huffman

Department of Chemistry and Molecular Structure Center, Indiana University Bloomington, IN 47405 (USA)

[**] Diese Arbeit wurde von der National Science Foundation der USA (Grant No. 83-05281) und vom Canadian Natural Sciences and Engineering Council gefördert. Wir danken den Firmen Johnson Matthey, Inc. und Rhenium Alloys, Inc. für Chemikalienspenden sowie Scont Horn für technische Unterstüzung. 